\title{
L'immigration : une affaire d'Etats. Conversion des regards sur les migrations algériennes (1961-1973)
}

Immigration: a matter of States. The transformation of perspectives

\section{Sylvain Laurens}

\section{(2) OpenEdition}

12 Journals

\section{Édition électronique}

URL : http://journals.openedition.org/conflits/10503

DOI : $10.4000 /$ conflits. 10503

ISSN : $1777-5345$

Éditeur :

CCLS - Centre d'études sur les conflits lilberté et sécurité, L'Harmattan

Édition imprimée

Date de publication : 20 avril 2008

Pagination : 33-53

ISBN : 978-2-296-05252-9

ISSN : 1157-996X

Référence électronique

Sylvain Laurens, «L'immigration : une affaire d'Etats. Conversion des regards sur les migrations algériennes (1961-1973)», Cultures \& Conflits [En ligne], 69 | printemps 2008, mis en ligne le 26 juin 2008, consulté le 30 mars 2021. URL : http://journals.openedition.org/conflits/10503 ; DOI : https:// doi.org/10.4000/conflits. 10503 


\section{L'immigration : une affaire d'Etats. Conversion des regards sur les migrations algériennes (1961-1973)}

\section{Sylvain LAURENS}

Sylvain Laurens est Maître de conférences en sociologie à l'université de Limoges. Chercheur an GRESCO (EA 3815) et an CURAPP (UMR 6054), ses travaux actuels portent sur les politiques d'immigration et les transformations de l'Etat dans les années suivant la décolonisation. Contact : sylvainlaurens@free.fr

C 'administration de l'immigration repose en France sur un mode de légitimation fondé sur la notion de "souveraineté » 1 et sur la distinction entre nationaux et étrangers. Elle est le produit d'une longue histoire de surveillance des populations étrangères sur le sol national. Historiquement, l'étranger est celui qui est exclu de la citoyenneté, soupçonné de ne pas faire preuve de «loyalisme » 2. L'Etat, par la mise en place progressive de techniques d'identification ${ }^{3}$, a cherché à contrôler ses déplacements d'abord à des fins politiques, puis pour distinguer la main-d'œuvre nationale de la main-d'œuvre étrangère sur le marché du travail (surtout à compter des premières grandes crises économiques de la fin du XIXe siècle et l'instauration du code de la nationalité en $1889{ }^{4}$ ). Ce mode de domination bureaucratique repose sur un lexique singulier lié notamment à l'enracinement de la IIIe République et à l'émergence d'un groupe de fonctionnaires spécialisés. L'administration coloniale repose, elle, sur un mode de légitimation fondé historiquement sur l'idée de « mission civilisatrice » : certains indigènes peuvent être des "nationaux ", mais ils sont exclus des droits politiques, «la négation de ces droits étant la condition de leur infériorisation sociale 5 ».

1. Beaud O., La Puissance de l'Etat, Paris, PUF, 1994, p. 111.

2. Lochak D., Etrangers, de quel droit ?, Paris, PUF, 1985.

3. Crettiez X., Piazza P. (dirs.), Du papier à la biométrie : identifier les individus, Paris, Presses de Sciences Po, 2006.

4. Rygiel P., Le Bon Grain et l'ivraie, l'Etat-nation et les populations immigrées 19e-20e siècles, Paris, Presses de l'ENS, 2006.

5 . Spire A., Colonisation et Immigration, communication au Congrès de l'Association française de sociologie, septembre 2006. 
Schématiquement, et si on s'attache uniquement à la question de l'encadrement administratif des flux migratoires, la décolonisation peut s'analyser dans les termes d'un passage progressif d'un mode de domination bureaucratique à un autre $^{6}$. Chaque processus d'indépendance a en effet suscité une série d'interactions entre les fonctionnaires-gouvernants français et les représentants de ces nouveaux Etats. C'est au terme de ces rapports de force successifs - dans le cadre desquels a été négocié un grand nombre de questions économiques, militaires mais aussi juridiques - que s'est joué le sort de ceux qui, d' "indigènes », étaient désormais appelés à devenir les «ressortissants » d'un pays étranger.

Dans un premier temps, la plupart des ressortissants des anciennes colonies se sont vus octroyer, au gré de ces négociations, des droits de circulation très étendus. Mais, dans un deuxième temps et dans la mesure où ce processus de décolonisation allait de pair avec une réévaluation des intérêts français en Afrique et l'avancée de la construction européenne, les agents du Quai d'Orsay et de bien d'autres ministères ont multiplié les négociations bilatérales avec ces pays nouvellement indépendants, dans le but de réduire ces droits de circulation, tandis que des droits toujours plus étendus étaient accordés aux pays de la zone européenne. Des accords ont ainsi été signés presque simultanément avec la Tunisie (commission mixte créée en 1963, accord du 11 décembre 1975), le Maroc (commission mixte créée en 1963, accords du 25 janvier 1978), le Portugal (accords de main-d'œuvre de 1963, 1971 et du 11 janvier 1977), l'Espagne (commission mixte créée en 1961, accords du 9 juillet 1976), l’Algérie (1962 et 1968), la Turquie (accords du 8 avril 1975), la Mauritanie (convention de circulation de 1963, négociations de 1976), la Haute Volta (convention de 1970), le Niger (1970), le Bénin (1971 et 1975), la Côte d'Ivoire (1970), le Mali (1963 et 1974), le Cameroun (1976) et le Sénégal (mars 1974). Ces accords ne sont que la face immergée et la concrétisation dans le droit d'interactions incessantes, voire de tentatives de renégociations parfois échouées qui visent invariablement à aligner les ressortissants de l'ancien Empire sur l'ordonnance de 1945 et à octroyer, à l'inverse, aux ressortissants européens des droits étendus de circulation.

Au fil de ces interactions, des flux migratoires (parfois très anciens) entre la France et les anciennes colonies ont ainsi été irrémédiablement saisis par une logique «interétatique 7 ».

Une analyse de cette transformation à grande échelle des rapports entre l'Etat français et tous les Etats nouvellement indépendants constituerait en soi un programme de recherche. A partir d'un travail sur archives consacré principale-

6. Cette distinction est moins établie si on se situe du côté du patronat métropolitain par exemple, qui cherche à combler un déficit de main-d'œuvre dès le début du $\mathrm{XX}^{\mathrm{e}}$ siècle sans s'arrêter à ces distinctions.

7. Nous empruntons le terme «interétatique » à Elias N., Engagement et distanciation, Paris, Fayard, 1983. 
ment aux hauts fonctionnaires français en charge de l'immigration ${ }^{8}$, cet article se limitera à l'exemple algérien, en analysant les interactions entre les hauts fonctionnaires métropolitains et les représentants du FLN au moment de l'indépendance. Nous montrerons comment les migrations entre la France et l'Algérie se sont trouvées saisies par ce processus multiforme d'étatisation et de transformation des modes de domination bureaucratique. La mise au jour de ce processus ne remet bien évidemment pas en cause les résultats des travaux démontrant, pour d'autres échelons administratifs, la reconduction, au-delà de la décolonisation, de pratiques d'encadrement issues du moment colonial 9 . Il s'agit d'introduire ici un point de vue complémentaire, qui permet de saisir toute la complexité d'une séquence historique où le redéploiement des agents de l'Etat impérial en métropole va de pair avec la construction de nouveaux régimes d'extranéité, reposant sur l'instauration de rapports diplomatiques bilatéraux avec les anciennes colonies. Seul un tel point de vue complémentaire peut permettre de comprendre comment la redéfinition des droits de circulation pour les anciens indigènes a conféré une acuité nouvelle à une logique interétatique, en faisant de ces mouvements de population une affaire d'Etat à Etat, une affaire d'Etats.

\section{Des migrations d'indigènes à « l'immigration algérienne "}

Lorsqu'il s'agit de désigner la façon dont les pouvoirs publics métropolitains appréhendent les «migrations algériennes » entre 1947 et 1962, la plupart des chercheurs utilisent alternativement et indifféremment les termes « migration » et « immigration ». Or, malgré nos recherches, à aucun moment les acteurs politiques ou les hauts fonctionnaires d'alors ne semblent employer le terme « immigration » au sujet des Algériens 10. C'est bien le terme « migration » qui est mobilisé. Alors que l'expression « immigration nord-africaine 11 » est parfois employée pour désigner les migrants tunisiens ou marocains, la perception des migrants algériens par le pouvoir parisien fait appel, avant 1962, à un autre lexique.

Il ne s'agit pas là de jouer sur les mots, mais de rappeler à quel point l'histoire particulière de la conquête de l'Algérie par la France s'est objectivée dans un dispositif politique et administratif singulier dont les catégories de classement diffèrent de celles usuellement employées à l'égard des étrangers.

8 . Laurens S., Hauts Fonctionnaires et immigration en France 1962-1982. Socio-bistoire d'une domination à distance, thèse pour le doctorat de l'EHESS, Noiriel G. (dir.), 2006. Idéalement, une telle mise en perspective nécessiterait d'explorer les fonds d'archives français et algériens. Or, cet article exploratoire mobilise essentiellement des fonds français (et, en complément, des témoignages publics d'anciens responsables du FLN).

9 . Se reporter à « La colonie rapatriée », Politix, $\mathrm{n}^{\circ} 76,2006$.

10. Le terme d' "immigration » naît sous la III' République et renvoie à la perception, par l'administration métropolitaine, des étrangers présents sur le territoire national.

11. Le terme d' "immigration » est utilisé par les services statistiques français pour désigner aussi les Français qui changeaient de résidence de la France vers l'Afrique du Nord. Dans ce même dénombrement de la population non musulmane en Algérie, le concept d'étranger est utilisé pour comptabiliser les populations venant d'autres pays européens (Espagnols, Italiens, Suisses, etc.). Voir Gouvernement général de l'Algérie, Service de statistique générale, vol 2, 1948, p. 16. 
Une analyse de la construction juridique d'une extranéité algérienne nécessite d'emblée, en effet, d'adopter une perspective diachronique. Pour le pouvoir parisien, parler d'« immigration algérienne » avant 1962 aurait signifié reconnaître un caractère extra-national aux "Algériens ", alors même que le discours officiel émis par les autorités contre le FLN (surtout à compter de 1958) était un discours d'inclusion. Pour l'historien, parler d' « immigration algérienne » avant 1947, c'est écarter d'un revers de main le fait que, de part et d'autre de la Méditerranée, un dispositif politico-administratif appréhende les déplacements de travailleurs algériens comme un flux d' «indigènes musulmans ». C'est écarter qu'en 1947, après quelques semaines d'hésitation sur l'alignement possible du «cas » algérien sur les logiques assimilationnistes (promues entre autres par un groupe de dirigeants démocrates-chrétiens) ${ }^{12}$, un consensus administratif s'était dégagé pour maintenir une distinction à l'égard des Français musulmans d'Algérie (FMA).

Mais surtout, parler d'« immigration algérienne » pour «l'avant 1962 » revient à occulter que les termes «immigration » ou «immigré » sont alors réservés en Algérie à une autre catégorie de population. Ils sont en effet exclusivement utilisés par l'administration pour désigner les «immigrés italiens » et autres «clandestins non nationaux $» 13$.

Si les mots de l'historien n'ont pas à être systématiquement ceux des acteurs historiques, il est probable que, sur ce point, nombre de travaux cèdent à l'anachronisme et soient victimes d'un voile téléologique qui conduit à raconter l'histoire des flux migratoires algériens avec le lexique du présent. Ralph Schor évoque ainsi «l'immigration algérienne » qui se «développa fortement» dans les années 1950 14, Vincent Viet évoque comment «l'immigration algérienne a bouleversé la politique de repopulation 15 » à la Libération et Benjamin Stora analyse « l'immigration algérienne en France entre 1912 et 199216 ».

Rappelons une nouvelle fois, dans le sillage de nombreux travaux récents, toute la particularité que revêtent, pour les autorités parisiennes, les flux des migrants algériens entre 1947 et 1962 17. Ni véritablement étrangers, ni véritablement citoyens français à part entière, les « travailleurs algériens » font l'ob-

12. Spire A., Sociologie historique des pratiques administratives à l'égard des étrangers en France (1945-1975), thèse pour le doctorat de sociologie, Suaud C. (dir.), université de Nantes, 2003, p. 144.

13. Centre des archives d'Outre-Mer (CAOM), versement 81 F 1589.

14. Schor R., Histoire de l'immigration en France de la fin du XIX $X^{e}$ siècle à nos jours, Paris, Armand Collin, 1996, p. 198.

15 . Viet V., Le Cheminement des structures administratives et la politique française de l'immigration (1914-1986), Paris, MIRE, 1996, p. 153.

16. Stora B., Ils venaient d'Algérie. L'immigration algérienne en France 1912-1992, Paris, Fayard, 1992.

17 . Spire A., "Semblables et pourtant différents. La citoyenneté paradoxale des Français musulmans d'Algérie en métropole », Genèses, n53, décembre 2003, p. 68. 
jet d'un dispositif à la fois juridique et administratif « à part » et dont la mise à bas est justement au cœur d'une discussion entre le FLN et les négociateurs métropolitains au moment des accords d'Evian.

Si, au lieu de désigner les migrants algériens d'avant et d'après 1962 par le même terme d' "immigrés », qui ne renvoie à rien sur le plan de l'activité administrative réelle de l'époque, on analyse « pas à pas » la genèse d'un nouveau statut pour les migrants algériens, les choses se compliquent pour le socio-historien. En effet, obtenir une vision claire des choses nécessite de tenir ensemble les différentes facettes d'un processus au terme duquel l'ensemble d'une population «française musulmane » se vit retirer ses papiers d'identité et attribuer, par la magie du droit, une nouvelle nationalité "à distance », tandis qu'au même moment, l'ensemble d'un groupe de Français «colons d'Algérie » devint, lui aussi par la force du droit potentiellement « étranger » sans avoir à migrer ${ }^{18}$. Si, selon les mots de Michel Offerlé, l'histoire est bien "un cimetière d'intérêts 19 ", le détour par les "processus inaboutis, possibilités qui, ouvertes dans certaines conjonctures, $n$ 'ont finalement pas été concrétisées 20 » permet d'analyser tout ce qui serait occulté si l'on réduisait le passé aux dichotomies claires du présent entre «Français » et « Algériens », « nationaux français » et «immigrés algériens ». Il apparaît alors que le statut particulier donné aux Algériens en France après 1962 et la mise en place des « certificats de résidence » (et non pas d'un système de cartes de séjour) à compter de 1968 sont bien le produit d'une confrontation continue entre deux groupes d'acteurs s'instituant progressivement en défenseurs et porte-parole de deux « Etats-nations » 21.

\section{"L'immigration algérienne": le sous-produit d'un cheminement sinueux vers deux Etats-nations séparés}

Le statut adopté à Evian est le produit altéré de projets sans cesse discutés et amendés visant à aménager un statut pour « une communauté européenne minoritaire », voire de projets envisagés avec plus ou moins de réalisme de découper l'Algérie afin de créer une enclave française. On ne peut comprendre comment un statut exceptionnel a pu être donné à compter de 1962 aux migrants algériens si l'on n'intègre pas à quel point le sort de la communauté algérienne (musulmane) en France s'est trouvé lié, pour les négociateurs d'Evian et les agents du Quai d'Orsay, au sort de la communauté européenne d'Algérie. Le statut à don-

18. Les enfants nés en Algérie des premiers colons peuvent refuser la nationalité algérienne en 1962 et, de ce fait, devenir des étrangers français dans l’Algérie indépendante sans jamais avoir vécu en France métropolitaine auparavant et sans avoir migré.

19. Offerlé M., Sociologie des groupes d'intérêt, Paris, PUF, 1994, p. 47.

20. Buton F., "L'Etat et ses catégories comme objets d'analyse socio-historique », in Laborier P., Trom D., Historicités de l'action publique, Paris, PUF, 2003, p. 59.

21. Ce point de vue sur les accords d'Evian est sensiblement différent de celui de T. Schepard in Shepard T., The Invention of Decolonization: The Algerian War and the Remarking of France, Cornell University, Ithaca, 2006. Nous renvoyons sur ce point à une recension à paraître dans Les Annales, Histoire, Sciences sociales, n³, 2008. 
ner à cette dernière constitue, dès 1961 (avec le sort du Sahara et les ressources pétrolières), l'un des enjeux principaux des négociations entre les représentants de l'Etat français et du FLN. Afin d'éviter un découpage territorial, les négociateurs métropolitains espèrent pouvoir parvenir à un découpage juridique qui maintiendrait la «communauté européenne d'Algérie » et les Français musulmans résidants en France dans un statut particulier.

Comme a déjà pu le souligner Sylvie Thénaut dans ses travaux, ces diplomates voudraient dans le cadre des accords d'Evian ériger les « Français d'Algérie » « en minorité protégée par des dispositions particulières 22 » et préserver ainsi leur influence dans le nouvel Etat algérien. Ces derniers espèrent empêcher un " exode massif de la population française » au moment d'une éventuelle indépendance en assurant un statut protecteur à une minorité européenne au sein d'un Etat algérien "qui non seulement apporterait [aux] nationaux la sécurité de leurs biens ou de leurs emplois, le libre exercice des libertés individuelles et des libertés publiques, mais qui instituerait aussi un système efficace de garanties collectives 23 ». Dans les documents préparatoires des accords d'Evian, le sort de la communauté musulmane algérienne en France semble donc intimement lié au sort de cette future « minorité européenne ». Selon ces fonctionnaires-gouvernants, dans ces négociations « la France » disposerait d'un argument de poids pour emporter l'adhésion des leaders indépendantistes vis-à-vis de ce statut de la minorité. Il suffirait de proposer aux leaders du FLN la réciprocité des droits :

«Il lui suffira [à la France] - pour ce faire - de proposer la réciprocité de traitement : il y a en France environ 400000 travailleurs algériens. On peut parfaitement envisager qu'ils jouissent sur le territoire de la métropole, dès lors qu'ils manifestent leur intention d'y séjourner quelques années, d'un certain nombre de droits et de privilèges analogues à ceux résultant du "statut de la minorité” applicable aux Français d'Algérie ${ }^{24}$. »

En 1961, ces hauts fonctionnaires espèrent toujours imposer un projet à deux facettes aux leaders du FLN. D’un côté, les "Européens d'Algérie », appelés selon les projets provisoires "Algériens minoritaires" ou «Algériens européens ", bénéficieraient d'une "part équitable à la gestion des affaires publiques, qu'il s'agisse des affaires générales de l'Algérie ou des affaires des collectivités locales, des établissements publics et entreprises publiques " ${ }^{25}$. Ils disposeraient d'une « authentique représentation 》 dans «toutes les assemblées à caractère politique, administratif, économique, social ou professionnel " en Algérie. Le droit constitutionnel algérien leur garantirait «dans chaque assem-

22. Thénault S., Histoire de la guerre d'indépendance algérienne, Paris, Flammarion, 2005, p. 243.

23. CAOM 81F/148. Cabinet du ministre d'Etat aux Affaires algériennes, mars 1961, note générale sur le statut des communautés.

24. Ibid.

25. Ibid. 
blée » une représentation importante et, pour tout litige, d'un juge issu de la même «communauté ».

De l'autre côté, et « en creux » du statut donné aux Européens, les «musulmans installés en France " ne "possèderaient pas la double nationalité et n'auraient pas droit à l'établissement d'un statut municipal dans les villes où ils habitent en forte proportion ", mais ces derniers "bénéficieraient de tous les autres droits ou garanties reconnus aux minoritaires en Algérie » 26 . Autrement dit, les «musulmans installés en France, ressortissants algériens » disposeraient notamment de la «jouissance de toutes les libertés individuelles et publiques ", du "libre accès aux emplois privés de toute nature ", ainsi que $\mathrm{du}$ "droit de fonder des écoles privées musulmanes » 27 . En cas de litige, ces derniers bénéficieraient de «l'application de leur statut personnel » et disposeraient du «droit d'être jugés par des tribunaux où siègerait un juge algérien ». Sur le plan municipal, symétriquement au statut défendu pour les Algériens européens en Algérie ${ }^{28}$, les conseillers de Louis Joxe prônent l'idée d'une "participation à la gestion municipale dans les villes où existe une population algérienne importante (plus de $10 \%$ )». Leur séjour ne serait pas soumis à la possession d'un titre de séjour mais à un «certificat d'établissement 29 » (équivalent du certificat de résidence, qui sera finalement adopté en octobre 1968). On le voit ici dans sa genèse même, les droits de circulation et les libertés octroyées aux travailleurs algériens en France sont pensés initialement par ces conseillers comme des contreparties cédées afin de préserver les intérêts et l'influence des «Européens d'Algérie ». Face à ces projets d'instaurer des communautés juridiquement protégées, les représentants du FLN vont opposer pendant toutes les négociations d'Evian l'idée d'une nation algérienne « une et indivisible » contribuant ainsi à l'affirmation d'un découpage juridique reposant exclusivement sur des bases étatico-nationales.

\section{Evian et les luttes autour du contenu d'une "nation algérienne »}

A quelques jours du lancement des négociations d'Evian, les négociateurs parisiens espèrent toujours obtenir un statut particulier et pensé sur des bases de réciprocité. Mais, dès le premier jour des négociations ${ }^{30}$, ce projet d'instaurer des minorités protégées rencontre une opposition franche de la part des négo-

26. CAOM 81 F/148. Note (non signée) de mars 1961 sur la réciprocité d'une garantie des minorités, cabinet de M. Joxe.

27. Ibid.

28 . Les accords prévoient que, dans toute commune algérienne où les Européens représentent plus de $10 \%$ de la population, un conseiller municipal européen siège d'office au conseil municipal.

29. Ibid.

30. CAOM 81 F 150. Procès-verbal de la première séance du 20 mai 1961, intervention du responsable de la délégation algérienne A. Belkacem : "Ce problème algérien est celui d'une décolonisation totale. Elle implique la disparition d'un système périmé et l'accession du peuple algérien à l'indépendance. [...] l'intégrité du territoire national et l'unité du peuple algérien doivent être respectées ». 
ciateurs du FLN, qui surprend les diplomates 31 et tempère leurs espoirs de parvenir à imposer leur point de vue dans l'accord final. Les négociations s'enlisent et, des mois durant, le sort des musulmans algériens installés en France va ainsi se trouver irrémédiablement lié, «à distance ", à un conflit autour de la notion de «nation ». Les représentants du FLN s'instituent en gardiens d'un Etatnation algérien en devenir et rejettent conjointement à la fois l'éventualité d'une partition administrative et l'idée d'une «minorité protégée » qui s'opposerait, selon eux, à l'idée de souveraineté du peuple algérien. Les débats se développent alors autour du contenu de la future «identité nationale» algérienne. Convoquant le thème de « la terre » et des «morts », les négociateurs parisiens défendent l'idée que cette nation ne pourrait être «une nation» sans intégrer ceux qui ont vécu sur son sol durant "plus d'un siècle» ${ }^{32}$. Louis Joxe plaide pour une "nécessaire » coexistence entre minoritaires et majoritaires au sein d'un même ensemble politique dont le contenu resterait à définir. Les procèsverbaux de ces échanges (dont la plupart sont retranscrits mot pour mot) montrent comment lce dernier, en citant Ernest Renan, définit la nation «comme un principe spirituel ", une " âme », et enjoint les responsables algériens à intégrer dans la nation à venir la communauté européenne sous un statut particulier, légitimant ce statut par l'idée qu'il s'agirait là, déjà, d'une «famille » soudée par une histoire commune et singulière :

«Le terme de "peuple” est juridiquement et politiquement imprécis. Aucun des éléments recherchés pour déterminer le contenu de cette notion n'est suffisant ou décisif. La géographie ne suffit pas, [...] ni la religion, ni la langue [...]. Le cumul de ces critères ne permet pas de parvenir à la notion de peuple. Il vaut mieux reprendre la définition de Renan, selon laquelle la nation est un "principe spirituel”, une "âme”; le souvenir des grandes actions faites ensemble, la volonté d'en entreprendre d'autres dans l'avenir. [...] A défaut, il n'y aurait hélas qu'une autre voie possible, c'est que les membres de la communauté européenne soient regroupés par la France avec les autres Algériens qui voudraient rester Français afin qu'ils puissent demeurer en Algérie et y vivre chez eux comme ils en ont le droit ${ }^{33}$. »

Contre cette définition d'une nation algérienne qui resterait à construire, contre cette idée d'une «nécessaire » détermination de droits distincts à l'intérieur d'une même nationalité, les leaders du FLN opposent systématiquement l'existence d'une nation algérienne déjà plus que centenaire et dans laquelle les anciens colons, «alluvions de l'histoire 34 », n'auraient qu'à se fondre sans statut particulier.

31. La délégation ne pouvait visiblement céder sur ce point de l'unité nationale au risque de subir des dissensions internes, voir le livre autobiographique de l'ancien président du GPRA, Ben Khedda B., Les Accords d'Evian, Paris, Sud/OPU, 1986.

32. CAOM 81 F 150. Intervention de Louis Joxe lors de la réunion du 25 mai 1961.

33. Ibid. Procès-verbal du 6 juin 1961, déclaration de la délégation française (L. Joxe).

34. Ibid. Procès-verbal de la séance du 10 juin 1961. Intervention de B. Krim (FLN). 
Ces longs échanges autour du contenu et des conditions d'accès de certains groupes à la nationalité algérienne montrent comment la sortie d'un système de domination bureaucratique colonial est passé systématiquement par un processus de redéfinition des ressorts de légitimité sur lesquels étaient fondés les découpages juridiques préexistants. Ces interactions nous permettent ici surtout de mettre au jour tout ce que le basculement d'un mode de légitimation à l'autre, loin d'être évident et « logique ", doit à ces rapports de force permanents entre, d'une part, des représentants des futurs Etats indépendants et, d'autre part, des « grands commis » qui se trouvent institués dans ces interactions en garants des droits d'une «communauté nationale» sur le point de devenir « étrangère » dans les anciennes possessions.

Si les droits de circulation accordés à ces nouveaux migrants furent distincts de ceux octroyés aux étrangers régis par l'ordonnance de 1945, c'est notamment parce que ces rapports de force n'aboutirent pas toujours «naturellement » et «spontanément » à un compromis. Ainsi, à Evian, après vingt et un jours de négociation, aucune avancée n'a pu être obtenue. Face à des négociateurs parisiens manifestement confiants au début des négociations, les représentants du FLN défendent toujours sans faillir l'idée d'un ensemble national un et indivisible, sans communauté intégrée en son sein qui disposerait de droits particuliers. Après les négociations des Rousses, un compromis est trouvé, à mi-chemin entre la conception d'un droit des communautés et la définition souveraine de la nation algérienne défendue par les représentants du FLN. Afin de préserver les «intérêts français » en Algérie, les diplomates s'accordent sur une liberté réciproque de circuler («sauf décision de justice tout Algérien muni d'une carte d'identité est libre de circuler entre l'Algérie et la France $35 »)$. La communauté européenne d'Algérie se voit désignée par son "statut civil de droit commun 36 » et découpée sous un double critère d'ancienneté de résidence en Algérie et de filiation. Au terme de trois ans, la nationalité algérienne est optionnelle et s'obtient par inscription sur les listes électorales.

L'exemple de l'Algérie est sans doute un exemple particulier, tant par les conditions de l'accession à l'indépendance que par l'importance de la colonie « européenne » installée et, donc, des enjeux économiques soulevés. Mais cet exemple est d'autant plus intéressant qu'il permet de voir, presque « en raccourci » et dans la continuité des événements, comment ces compromis passés au moment des indépendances ont pu être remis en cause en seulement quelques années.

35. Les Accord d'Evian, op. cit., p. 37.

36. Dans les accords d'Evian définitifs, la « communauté européenne » est définie ainsi : «les Français quelles que soient leur origine ou leur religion qui relèvent du statut civil de droit commun par opposition au statut civil local ou coranique». 


\section{Les migrations franco-algériennes saisies par des rapports entre Etats-nations}

A compter du moment où deux entités bureaucratiques définies sur des bases nationales se font face, les flux de personnes entre la France et l'Algérie se trouvent saisis, de part et d'autre de la Méditerranée, par des rapports diplomatiques incessants. Le cessez-le-feu est conclu le 19 mars. N'ayant pu parvenir à arracher un statut juridique hybride pour la colonie européenne, le 3 juillet, le gouvernement français envoie son premier ambassadeur et des consuls «partout où l'importance de la colonie française justifie sa présence 37 ». Témoignant de l'enjeu particulier que représente la « question algérienne » pour le pouvoir politique et au sein de l'Etat, le général de Gaulle nomme lui-même comme premier ambassadeur de France à Alger un de ses anciens ministres: Jean-Marcel Jeanneney ${ }^{38}$. En quelques semaines, le Quai d'Orsay met en place une représentation consulaire dans chacune des villes où la colonie "européenne »devenue potentiellement binationale par la magie du droit - est installée. Le fonds d'archives déposé par Jean-Marcel Jeanneney permet de mesurer comment s'opère, dans la continuité des accords d'Evian et en seulement quelques semaines, une conversion du regard de part et d'autre de la Méditerranée sur les flux de personnes entre la France et l'Algérie, tant depuis le point de vue du gouvernement français que du nouveau gouvernement algérien.

\section{"Protéger nos ressortissants » : l'intérêt des hauts fonctionnaires français à l'étatisation de la société algérienne}

A compter de septembre 1962, les gouvernants algériens deviennent en quelques mois, pour les fonctionnaires-gouvernants français, les interlocuteurs diplomatiques incontournables par lesquels se décide du sort de 312000 ressortissants français (devenus) expatriés ${ }^{39}$, auxquels est liée la préservation d'intérêts économiques et stratégiques. Tandis que, réciproquement, les conditions de séjour de 350000 étrangers algériens en France se voient tout autant déterminées, du côté algérien, par les relations bilatérales instaurées avec l'ancienne puissance coloniale. Il est saisissant de voir à quel point les relations franco-algériennes, à partir de l'hiver 1962, deviennent en seulement quelques semaines des relations d'Etat à Etat. Jusque dans leurs formes protocolaires, les échanges sont désormais régis par le cadre structurant des échanges diplomatiques. Il est également frappant de voir dans quelle mesure le processus d'étatisation de la société algérienne est encouragé par les représentants français. L'équipe consulaire participe, en effet, à

37. Fonds Jean-Marcel Jeanneney (JMJ). Compte rendu «très secret» du conseil des Affaires algériennes, rapport de J.-M. Jeanneney du 16 novembre 1962.

38 . Né en 1910, fils de Jules Jeanneney, ancien président du Sénat, professeur agrégé de droit, il débute sa carrière politique auprès de son père, ministre à la Libération. Ministre de l'Industrie (1958-1959), Jean Marcel Jeanneney qualifie d' inéluctable » l'indépendance algérienne au moment du Conseil des ministres d'août 1959 consacré à cette question.

39. Fonds JMJ. Télégramme de l’ambassadeur de France à Alger, août 1962. 
la délimitation des contours d'un nouvel Etat: Trésors publics, Sécurités sociales, comptabilités nationales sont séparés, les règles des relations commerciales et de coopération militaire sont établies avec l'aide des diplomates français. Loin des représentations qui pourraient être portées par des acteurs mémoriels qui limitent l'histoire de la décolonisation aux départs des pied-noirs, la préservation des intérêts «français » passe pour ces hauts fonctionnaires, dès le lendemain de l'autodétermination, par la mise en place de relations bilatérales soutenues. Proche en un sens de ce qu'un observateur extérieur pourrait qualifier de Real Politik, les responsables français souhaitent non pas un «effondrement algérien », synonyme d'une revanche historique, mais la mise en place la plus rapide possible d'un Etat « viable » afin de disposer d'interlocuteurs stables.

L'intérêt français à l'étatisation de la société algérienne a deux moteurs principaux. Si le premier est économique, lié essentiellement aux ressources pétrolières ${ }^{40}$, le second - et c'est sans doute ce qui nous intéresse plus particulièrement ici - a trait plus spécifiquement au sort des ressortissants français. Très vite en effet, les diplomates français perçoivent tout l'intérêt d'une bureaucratie stable et d'un ordre public rétabli pour assurer la « sécurité » de leurs ressortissants. Dans un courrier adressé au gouvernement, le nouvel ambassadeur s'inquiète des exactions dont lui semblent victimes les ressortissants français. Cette « réaction physiologique » du peuple algérien conduisant à «l'expulsion d'un corps étranger » serait due notamment à «l'anarchie politique et administrative » et à «la faiblesse des moyens » du nouvel Etat algérien ${ }^{41}$. Si le lien intime entre «Etat » et «immigration » a déjà pu être pointé dans bien des travaux ${ }^{42}$, il prend ici un sens particulier. Tout se passe comme si la présence d' «immigrés français » en Algérie devait constituer, du côté des hauts fonctionnaires français, le carburant privilégié d'un encouragement à l'étatisation de la société algérienne. Il apparaît des échanges entre le quai d'Orsay et l'ambassade de France à Alger dans quelle mesure les autorités françaises sont inquiètes du devenir de ceux qui sont alors avec récurrence désignés comme des «compatriotes » ${ }^{43}$, mais aussi dans quelle mesure toute amélioration future ne pourra venir, que d'un renforcement de la bureaucratie algérienne. De ce fait, dans l'entre-soi des réunions à l'Elysée et à Matignon, les hauts fonctionnaires défendent l'idée que «la France a intérêt à ce que l'Etat algérien ne tombe pas dans la misère ni l'anarchie 44 ». Mais le départ

40. Fonds JMJ. L’indépendance est proclamée en 1962, mais la France, via ses entreprises nationales, reste le premier exploitant du pétrole algérien (celui-ci n'est nationalisé qu'en 1972 par H. Boumediene). Alors même que l'Algérie vient de déclarer son indépendance, des troupes françaises assurent la surveillance des installations pétrolières afin de « s'opposer par persuasion et à défaut par la force à toute intrusion de l'armée algérienne ou de tout élément armé d'origine algérienne sur les installations pétrolières » (télégramme «très secret » rédigé en novembre 1962 par le Premier ministre Georges Pompidou à l'attention du ministre des Armées).

41. Fonds JMJ. Rapport de l'ambassadeur de France à Alger, 7 novembre 1962.

42 . Noiriel G., Etat, Nation et immigration, vers une histoire du pouvoir, Paris, Belin, 2001.

43 . En effet en quelques jours le terme d' «Européens » disparaît.

44 . Fonds JMJ. Compte rendu «très secret» du conseil des Affaires algériennes du 16 novembre 1962. 
continu de ceux qu'il est désormais convenu d'appeler «les ressortissants français » va modifier très vite les termes de ces échanges diplomatiques.

La noblesse d'Etat face à la "défrancisation » et à l'émigration des "nationaux français »

Alors qu'en juin 1960, 1024800 «Européens » résidaient en Algérie, ils ne sont plus que 312000 en août 1962 45. Les représentants consulaires français sont les témoins privilégiés de cette migration de leurs ressortissants et les télégrammes envoyés quotidiennement au Quai d'Orsay se font de plus en plus alarmistes : "Une Algérie nouvelle est née, plus vite qu'on ne le pensait, et la place de nos compatriotes n'y sera pas aussi importante qu'on aurait pu le croire il y a quelques mois 46 ».

Lors du conseil des Affaires algériennes du 16 novembre 1962, les « sommets de l'Etat français » ne peuvent qu'acter de ces transformations profondes $\mathrm{du}$ contexte algérien. Les premiers entretiens « diplomatiques » entre Etat français et Etat algérien portent alors sur les « attentats contre les Européens » et sur l'expulsion de ceux qu'il est désormais convenu d'appeler, afin de lever toute ambiguïté, "des nationaux français 47 ». Peu à peu, à mesure que la «communauté européenne » quitte l'Algérie, il apparaît avec évidence aux représentants consulaires que la réciprocité et les clauses particulières obtenues à Evian ont perdu tout leur sens. L'exemple algérien illustre ainsi parfaitement comment l'évolution de la position des hauts fonctionnaires à l'égard des anciens indigènes issus des anciennes colonies dépend pour une part importante de l'évolution de ce qu'ils considèrent être les «intérêts français » en jeu. A l'aune des départs français et dans un mouvement presque mécanique, l'intérêt que les autorités françaises se devaient de porter aux départs d'Algériens vers la France est fortement réévalué. Le 7 novembre 1962, quelques jours seulement après l'autodétermination, l'ambassadeur français en Algérie fait longuement le point sur la situation à l'attention de son ministre de tutelle et du président de la République. Il explique dans un long rapport dans quel sens la ligne diplomatique française devrait être révisée. Selon lui, " cinq semaines après la constitution du premier gouvernement algérien, l'Algérie présente une situation sensiblement différente de celle qui avait été envisagée à Evian » et le "départ massif des Européens » fait perdre toute validité aux clauses prévoyant la participation politique de ces derniers aux institutions algériennes ${ }^{48}$.

45. Fonds JMJ. Télégramme du 27 août 1962 de l'ambassade d'Alger au Quai d'Orsay.

46. Ibid.

47. Fonds JMJ. Note du 26 novembre 1962.

48. Fonds JMJ. Rapport de l'ambassadeur de France à Alger, 7 novembre 1962. 
Du 1er septembre 1962 au 11 novembre 1962, des milliers d'Algériens 49 quittent l'Algérie pour la France. Dès les premières notes rédigées par les conseillers de l'ambassade, on perçoit comment les prévisions des accords d'Evian furent remises en cause tant pour la « communauté européenne » que pour la migration algérienne. Les droits de réciprocité étendus donnés aux migrants algériens en France avaient été octroyés dans l'idée que la communauté européenne d'Algérie resterait relativement nombreuse, or elle se voit réduite à la portion congrue en quelques semaines.

Cette symétrie des droits avait été concédée, en outre, en pensant que les migrants algériens ne resteraient que pour «un séjour temporaire». Or, "l'après-indépendance » correspond subitement, selon ces hauts fonctionnaires, à une «transformation des flux ». Non seulement les départs ne semblent plus être temporaires, mais ils semblent de plus en plus nombreux. On trouve, dès ces premières semaines et dans ces toutes premières notes, la matrice d'un nouveau discours diplomatique. Comme ce conseiller de l'ambassade de France à Alger en semble persuadé, ces départs seraient le fait de «travailleurs non qualifiés " à "l'état sanitaire douteux » et seraient intimement liés «à l'appauvrissement des villes $d u$ fait $d u$ départ des Européens $» 50$.

Alors qu'ils ont été les opérateurs d'un processus liant le sort de deux communautés lors des accords d'Evian, les diplomates français sont, de la même façon, ceux qui opèrent une réévaluation de l'intérêt qui devrait être porté à la migration algérienne. A l'issue du Conseil des Affaires algériennes du 16 novembre 1962, il est «acté » que ce nouvel «afflux migratoire » risque de «poser à la France des problèmes de maintien de l'ordre et d'organisation du marché du travail $\gg 51$. Cette mise en alerte rencontre les préoccupations des agents du ministère de l'Intérieur chargés de surveiller les arrivées de ces mêmes migrants.

\section{La duplicité des lexiques au sein du ministère de l'Intérieur}

En France, l'arrivée des travailleurs algériens est saisie au sein du ministère de l'Intérieur par deux dispositifs administratifs. D'une part et jusqu'en 1966, les structures en charge des Français musulmans d'Algérie (FMA) (devenus travailleurs algériens) sont toujours en activité. En région parisienne, le Service des affaires musulmanes (rebaptisé un temps SLPM ${ }^{52}$ ) et les Conseillers tech-

49. 91744 entrées pour 45000 sorties selon les chiffres donnés par Stora B., Ils venaient d'Algérie, op. cit., p. 144.

50 . Fonds JMJ. Télégramme du conseiller à l'ambassade de France, Guiringaud, à l'ambassadeur en déplacement à Paris, intitulé « Modification dans la composition du courant migratoire » et daté du 3 novembre 1962.

51 . Fonds JMJ. Conseil des affaires algériennes, le 16 novembre 1962.

52 . Service de liaison et de promotion des migrants. 
niques aux affaires musulmanes (CTAM) au sein des préfectures font alors écho aux rapports alarmistes des diplomates français. D'autre part - et du fait même que les migrants algériens sont désormais les ressortissants d'un Etat étranger - les mêmes migrants sont aussi surveillés par les agents des bureaux de la circulation transfrontière en charge de tous les étrangers.

Les archives déposées par le ministère de l'Intérieur à Fontainebleau permettent de se faire une idée de cette cohabitation de deux circuits administratifs disposant chacun de leur histoire et de leur lexique propres et qui mettent en alerte le pouvoir politique à leur façon. D'une part, les préfectures et le Service des affaires musulmanes (SAMAS) constituent, dès les premières semaines de l'indépendance, l'épicentre d'une appréhension de l'arrivée des migrants algériens avec la terminologie usitée avant l'indépendance. Les ressortissants algériens apparaissent donc dans les notes comme des «travailleurs musulmans 53 » ou des « oisifs 54 ». Dans une note du 30 novembre 1962 adressée à sa direction, Gilles Lamassoure (directeur du SAMAS), enjoint ainsi ses supérieurs de prendre des mesures face à un «mouvement migratoire en provenance d'Algérie 55 ». Comme le décrit avec précision Alexis Spire, au niveau des préfectures, le même personnel, toujours en place, conduit des «opérations de terrain » reprenant les techniques élaborées avant l'indépendance et procède à un nombre important d'expulsions ${ }^{56}$.

Mais, à peu près au même moment 57 , les hauts fonctionnaires situés au niveau de la sous-direction des étrangers et de la circulation transfrontalière mobilisent, quant à eux, un tout autre lexique. Ils considèrent ces derniers comme des «travailleurs algériens » et parlent également d'un «problème» mais, cette fois-ci, dans les termes d'un «mouvement d'immigration algérienne trop important 58 ».

Peu à peu, les rapports de force internes au ministère de l'Intérieur jouent en la faveur de l'imposition de ce dernier lexique. Le Service des affaires musulmanes est situé au contrebas des hiérarchies administratives (menacé de disparition et effectivement supprimé en 1966). Il dispose d'un maillage important sur tout le territoire via les CTAM, mais il n'est sans doute pas en mesure d'imposer son propre lexique face à la sous-direction des étrangers. Au fur et à mesure que l'on s'élève dans la hiérarchie et que l'on s'éloigne de la date d'indépendance, le lexique étranger/national est bien celui qui s'impose. La dichotomie national/étranger prédomine avec d'autant plus de force au sein du ministère de l'Intérieur, et plus largement au sein de l'Etat, que la solution à ce qui est bien désigné comme «un problème de l'immigration algérienne » ne peut désormais être trouvée qu’à

53. Centre d'archives contemporaines (CAC) 1989 0519, art. 28. Note de Gilles Lamassoure, 30 novembre 1962.

54. Spire A., op. cit., p. 208 et suivantes.

55. CAC 1989 0519, art. 28., op. cit.

56. Ibid.

57 . Pour être exact, deux jours avant la rédaction de la note citée ci-dessus.

58. CAC 1989 0519, art. 28. Note de la sous-direction des étrangers du 28 novembre 1962. 
travers une décision « diplomatique » : soit la signature d'une convention avec l'Algérie afin d' "organiser cette immigration algérienne » 59, soit une décision qui sera perçue sur le plan international comme « unilatérale».

\section{L'inscription des flux migratoires algériens dans une équation diplomatique plus large}

A compter de 1963, les agents du ministère de l'Intérieur essaient de façon récurrente de persuader les conseillers de leur ministre de tutelle et surtout les agents du Quai d'Orsay de la nécessité de procéder à un aménagement du statut des migrants algériens. Mais, dans le cas de l'Algérie, un changement des textes régissant ce statut tout juste négocié nécessite désormais une re-discussion des accords d'Evian (ce qui ne manquerait pas d'avoir une portée symbolique importante quelques mois seulement après leur signature). Plus encore, toute discussion concernant le sort des anciens indigènes devenus ressortissants algériens nécessite désormais de remettre sur le tapis diplomatique toute une série de questions connexes (pétrole, présence militaire en Algérie, statut de la mosquée de Paris, etc.).

Ainsi, même si la sortie de ces régimes de circulation spécifiques hérités de la décolonisation et l'alignement progressif sur l'ordonnance de 1945 des migrations issues de ces anciennes colonies constituent l'objectif principal que se fixent ces agents des ministères d' " encartement » dans ces années-là, les restrictions à la libre circulation des ressortissants des anciennes colonies ne va pouvoir s'effectuer que par étapes. L'inscription des flux migratoires algériens dans une équation diplomatique plus large ne rend possible des aménagements juridiques qu'au gré des « contradictions » propres au champ administratif français, et du souhait ponctuel des dirigeants algériens d'encadrer autant que faire se peut les déplacements de leurs propres ressortissants ${ }^{60}$. Là où les agents de l'Intérieur étaient en quelque sorte seuls responsables du sort des musulmans algériens en métropole avant 1962, le sort des « immigrés algériens " s’inscrit désormais dans un tout autre espace de discussion.

Saisi par des rapports interétatiques, tout ce qui les concerne est désormais du ressort d'agents du Quai d'Orsay et d'agents situés à des niveaux hiérarchiques bien plus élevés que ne l'étaient, par exemple, les structures policières d'encadrement des FMA. La question de ce qu'il est désormais convenu d'appeler «l’immigration algérienne » est traitée directement dans des échanges entre

59. CAC 1989 0519, art. 28. Note rédigée le 13 avril 1963 par le directeur de cabinet du ministre de l'Intérieur.

60. Du côté algérien, s’établit également dès l'indépendance un rapport coût/avantage autour de l'émigration vers la France. Pour les dirigeants algériens, les migrants présents en France constituent à la fois une aubaine financière (par l'importation de devises qu'ils suscitent), mais aussi un élément à intégrer dans une politique de l'emploi. L'émigration peut être à la fois un moyen d'exporter une partie du chômage et, à terme, un moyen de rapatrier dans l'économie algérienne des « cadres » formés en France. 
ministres, Premiers ministres, voire entre présidents des deux pays. Les hauts fonctionnaires investis dans ces interactions interviennent de plus en plus fréquemment dans un espace administratif de l'immigration, contribuant ainsi, parallèlement à son « ennoblissement 61 ».

Signés en 1964, les accords Nekkache-Grandval 62 symbolisent ce basculement historique par lequel le sort des migrants algériens est désormais inscrit dans de nouveaux " circuits » administratifs, ou plutôt au sein de ce que nous pourrions qualifier (en nous inspirant d'une sociologie des configurations) de nouvelles chaînes d'interdépendances administratives.

Les migrations post-coloniales inscrites dans de nouvelles chaînes d'interdépendances administratives

Penser en termes d'interdépendances nous permet ici de rappeler, en reprenant la métaphore de l'échiquier chère à Norbert Elias, dans quelle mesure chaque «coup » joué par les fonctionnaires-gouvernants français en direction des travailleurs algériens suscite désormais en réaction une évolution de la position algérienne. Ainsi, il devient extrêmement hasardeux de penser les décisions prises de part et d'autre en faisant fi de cette matrice de rapports sociaux, de ces interactions incessantes entre les agents de ces deux dispositifs bureaucratiques. Chaque décision, de la plus anodine à la plus spectaculaire, peut potentiellement être prise en réaction à une autre décision.

Encadré $n^{\circ} 1$ - Les relations bilatérales « égales en droit, inégales en fait »: apports et limites du point de vue d'Abdelmalek Sayad

Penser l'immigration dans ce qu'elle doit au travail effectué par les agents d'un Etat-nation ou aux rapports entre Etats n'est pas une idée nouvelle. Elle a notamment déjà été avancée par Abdelmalek Sayad qui rappelle à plusieurs reprises dans ses travaux comment l'Etat « fabrique » du national en « fabriquant » des étrangers ${ }^{63}$. Pour ce dernier, les transactions entre Etats ne sont néanmoins "bilatérales qu'en droit » et "décidées en réalité unilatéralement par le partenaire dominant » ${ }^{64}$. Analyser ces relations sous I'angle d'une relation de domination totale ne peut cependant que conduire à occulter un certain nombre de réalités. Certes, dans le cas par exemple des accords franco-algériens de 1964, "les concessions consenties des deux côtés ne sont pas du même ordre » et peuvent

61. Laurens S., Hauts Fonctionnaires et immigration en France, 1962-1982, op. cit.

62. Des noms respectifs des ministres du Travail algérien et français.

63. Sayad A., «Immigration et pensée d'Etat », Actes de la recherche en sciences sociales, 1999, n¹29, pp. 5-14.

64 . Sayad A., L'Immigration ou les paradoxes de l'altérité, Paris, De Boeck, 1991, p. 261.

65 . Ibid., p. 273. 
illustrer « I'état différentiel du rapport de force entre un pays dominant et un pays dominé » 65 . Mais il apparaît à l'analyse de ces rapports entre Etats que cette relation inégale ne se traduit jamais par une domination absolue et par l'absence de marges de manœuvre du côté du pays « dominé ». Si nous nous inscrivons dans la continuité des apports d'une sociologie de la domination, certains éléments seraient inéluctablement occultés si l'on ne prenait pas en compte deux dimensions essentielles de ces négociations.

Tout d'abord, le pays « dominant » ne contrôle que dans une certaine mesure les migrations et il y a toujours un écart important entre ce qui est souhaité par les fonctionnaires-gouvernants et la réalité des entrées sur le territoire. Or, pour Abdelmalek Sayad, la France serait en situation de " choix», inscrite dans un système de lowest bidder ${ }^{66}$. Elle aurait "le monopole presque exclusif du marché du travail pour immigrés ». La "France », acteur parfois réifié et sans contradictions internes, aurait une "totale maîtrise, c'est-à-dire tout à la fois le choix des partenaires et le contrôle des offres ou des quantités, des échéances, des enchères", afin d'obtenir la " main-d'œuvre disponible au moindre "prix" » 67. Cette théorie " du choix » nous semble devoir être fortement amendée. Tout d'abord, il est sans doute faux d'avancer l'idée que plus un pays serait "pauvre et dominé ", plus serait " avantageuse, sous tous les rapports, I'immigration originaire de ce pays " car l'intérêt d'une « immigration " pour " un pays » ne se limite pas à son seul prix sur le marché du travail, mais pose aussi la question (tout aussi cynique) de sa productivité et de sa qualification. Ensuite les politiques de « sélection » se heurtent toujours aux logiques sociales à l'origine des départs. Aucun Etat ne dispose sans doute des moyens de contrôler totalement les déplacements de population. Enfin, les pays "dominés " peuvent aussi avoir un intérêt à un contrôle des départs et un intérêt à l'émigration.

D'autre part, considérer, comme Abdelmalek Sayad, que ces négociations de main-d'œuvre ne sont «bilatérales que dans les formes " et « réciproques $q u$ 'en théorie " 68 confine à un certain anti-juridisme. Car le pays " dominé » dispose bien souvent de ressources - mêmes réduites - dans ces échanges. Ses représentants peuvent toujours faire valoir d'éventuels marchés économiques, la présence réciproque de ressortissants sur leur propre territoire... Ceux-ci disposent aussi, et surtout, de ressources plus spécifiquement politiques, de par l'organisation de la communauté expatriée dans des associations culturelles, par la presse ou la prise à partie de l'opinion publique du pays « dominant ». Ils peuvent aussi jouer sur les marges de manœuvre entrevues dans les instances internationales. Certes, ces marges de manœuvre ne remettent pas fondamentalement en cause l'inégalité de la relation, mais elles peuvent parfois contraindre les velléités des pays importateurs de main-d'œuvre. Dès lors, il apparaît

66. Enchères «négatives » où les fermiers américains embauchaient la main-d'œuvre la moins disante aux moments des crises économiques au XIX ${ }^{\mathrm{e}}$ siècle.

67 . Ibid., p. 263.

68 . Ibid., p. 267. 
toujours préférable, en la matière, de lier systématiquement une analyse en termes de domination à une analyse en termes d'interdépendances. Non pas pour substituer la seconde à la première, mais pour pouvoir intégrer tout ce qui contraint également le pays « dominant» dans ces relations bilatérales.

Le « contingent » des travailleurs algériens, c'est-à-dire le nombre des ressortissants algériens qui se verront octroyer le droit d'émigrer en France devient, à compter des accords Nekkache-Grandval de 1964, un objet récurrent de discussions parmi les diplomates des deux pays. La question de l'émigration-immigration des travailleurs algériens se trouve alors inscrite irrémédiablement, de part et d'autre, dans une équation complexe d'enjeux économiques, militaires et politiques. Dans ces relations bilatérales, les représentants algériens disposent de certaines ressources. A compter des accords de 1964, ils jouent notamment sur l'expiration des délais prévus par les accords précédents pour renégocier à partir du régime de libre circulation établi à Evian. Ainsi, en 1967, et ayant bien compris que les agents du ministère de l'Intérieur français craignent plus que tout un retour à un régime de libre circulation, ces derniers « jouent la montre ». A tel point que, à l'expiration des délais prévus par les accords de 1964, seuls les accords d'Evian s'appliquent officiellement.

L'accord d'octobre 1968 prévoit que les travailleurs algériens verront leur séjour encadré par l'instauration d'un certificat de résidence. Sur le plan des pratiques administratives, cet encartement correspond alors statistiquement à une décrue du nombre des expulsions (qui retrouvent leur niveau de 1963), et chronologiquement à l'extinction de l'ancien Service des affaires musulmanes. Produit de cette « bi-latéralisation » définitive, l'accord de 1968 prévoit également qu'un contingent de 35000 travailleurs algériens sera autorisé à venir en France chaque année pour une durée de deux ans. Sur le même modèle, cet accord court jusqu'en 1973, date de l'arrêt de l'émigration par l'Algérie.

$\mathrm{Si}$, au niveau de la préfecture de police et des opérations de terrain, cette période de transition peut se traduire par une reconduction de certaines pratiques coloniales, la même période, perçue depuis les sommets de l'Etat, correspond au triomphe d'une logique interétatique et d'un autre rapport de pouvoir. Chaque décision, prise par les fonctionnaires-gouvernants français ou les dirigeants algériens, doit désormais être pensée comme le produit de ces interdépendances complexes et ne peut être réduite à ses seules justifications publiques. Si l'on adopte ce point de vue, l'arrêt de l'émigration algérienne prononcé en 1973 peut être resitué dans sa complexité et dans ce qu'il doit notamment à une tentative du ministère de l'Intérieur de forcer la main au Quai d'Orsay et aux représentants algériens ${ }^{69}$.

69. Voir l'encadré nº 2 , page suivante. 
Ainsi, si l'on intègre cette nouvelle interdépendance entre deux ensembles bureaucratiques, l'arrêt de l'émigration algérienne vers la France, prononcé officiellement le 19 septembre 1973 par le président Boumédienne, peut faire l'objet d'une analyse qui diffère sensiblement des travaux historiques précédents. Si Yvan Gastaut explique, par exemple, cette décision par le « mécontentement " du président algérien face aux actes racistes commis lors de l'été $1973{ }^{70}$, du point de vue de ces rapports incessants entre Etats, il apparaît qu'une telle analyse revient à réduire les fondements d'une décision politique à sa seule légitimation publique.

A l'aune de ces rapports entre Etats, I'arrêt de l'émigration algérienne s'explique notamment en partie par une anticipation par le président algérien $d^{\prime}$ une décision unilatérale que comptait prendre le ministère de I'Intérieur français à l'égard des ressortissants algériens entrés sur le territoire français sans certificats de résidence. Dans le sillage des opérations de régularisation menées suite aux circulaires Marcellin-Fontanet (1972), les agents de la place Beauvau projettent de régulariser 6000 travailleurs algériens en situation irrégulière en retranchant ce nombre de 6000 du contingent des travailleurs autorisés à entrer en France pour l'année 1973. Le ministère de I'Intérieur presse ainsi pendant des semaines l'ambassadeur de France en Algérie de prévenir les représentants algériens de ce projet ${ }^{71}$.

L'ambassadeur français à Alger s'oppose d'abord à une telle idée, craignant les réactions de ses interlocuteurs 72 mais, ne disposant que d'une marge de manœuvre limitée, celui-ci obtempère le 19 septembre 1973, le jour même où le président Boumédiène doit clôturer le sommet des non-alignés à Alger. Le télégramme qu'il envoie à $15 \mathrm{~h} 15$ depuis I'ambassade éclaire alors sur la décision annoncée par le président algérien un peu plus tard dans la soirée :

« Extrêmement surpris par l'importance du nombre des travailleurs algériens en situation irrégulière, $M$. Benouniche [l'interlocuteur algérien] parlant à titre personnel, n'a pas caché ses préoccupations devant la mesure envisagée par les autorités françaises. Imputer ces 6000 travailleurs sur le contingent de l'année en cours reviendrait en effet à bloquer toute émigration jusqu'à la fin de 1973 et à annuler les départs d'ores et déjà prévus. Il y avait là, en tout état de cause, un problème sérieux qu'il convenait d'examiner et de résoudre d'urgence d'un commun accord.

70. Gastaut Y., L'Immigration et l'opinion en France sous la Ve République, Paris, Le Seuil, 2000, p. 29.

71. MAE 288 A 38 31. Note du 23 août 1973 du ministère de l'Intérieur.

72. MAE 288 A 38 31. Télégramme de l'ambassadeur au ministère de l'Intérieur du 20 août 1973.

73. MAE 288 A 38 31. Télégramme du 19 septembre 1973 (15h15) au Quai d'Orsay et ministère de l'Intérieur. 
Manifestement pris au dépourvu, M. Benouniche a promis de consulter dans les plus brefs délais les autorités intéressées et de nous faire part aussitôt de leur réaction ${ }^{73}$. »

Plutôt que de voir publiquement le gouvernement français amputer unilatéralement le contingent à venir pour l'année 1973 dans sa totalité, le président Boumédiène préfère exploiter cette opportunité au moment où le sommet des non alignés s'achève à Alger et où cette décision peut être considérée comme une « bravade " à l'égard de l'ancienne puissance coloniale. La suspension de toute émigration algérienne "jusqu'à nouvel ordre", cette décision prise quatre heures plus tard par le président Boumédiène au nom des «crimes racistes " dont sont victimes les travailleurs algériens s'explique structurellement par un nombre complexe de variables. Elle est, bien entendu, notamment encouragée par le contexte international qui place les dirigeants du FLN sur le devant de la scène au moment du sommet d'Alger, mais précisons surtout ici qu'elle ne peut être comprise que si on la réinscrit dans cette interdépendance continue entre ancienne puissance coloniale et pays nouvellement indépendant.

La décolonisation a induit la plupart du temps la sortie de régimes juridiques spéciaux et l'octroi de droits de circulation, de séjour et d'accès à la nationalité déconnectés de l'ordonnance de 1945. Ces droits spéciaux ${ }^{74}$ étaient bien souvent concédés comme une contrepartie du maintien d'une influence française dans les anciennes colonies. Si, dans le cas de l'Algérie, les droits de libre circulation et de réciprocité envisagés sont dans un premier temps étendus, cela est dû avant toute chose à l'importance numérique initiale de la colonie européenne et des intérêts économiques en présence. Mais l'exemple algérien prouve aussi dans quelle mesure les représentants diplomatiques participent au-delà des seules négociations d'indépendance à l'évaluation d'un rapport coût/avantage autour des flux migratoires. Dans le cas de l'Algérie, l'intérêt des représentants français au maintien de ces droits de circulation va évoluer très rapidement dans les années qui suivent la décolonisation. Il change en fonction du ralentissement économique et, parfois de la redéfinition des intérêts français dans la région.

Limiter l'analyse des pratiques administratives à l'égard des étrangers aux seuls ministères d'encadrement, sans intégrer ce qui se «joue » au même moment aux sommets de l'Etat, mais surtout dans les interactions entre Etats, ne permet pas de prendre en compte l'évolution de ces rapports de domination à grande échelle.

74. Qui sont également le produit de la situation antérieure, de la libre circulation à l'intérieur de l'ex-Empire français et des projets échoués d'établir une « citoyenneté impériale ». 
Comme le conseillait Norbert Elias ${ }^{75}$, le travail sociologique ne peut s'en tenir aux découpages académiques qui laisse aux seuls spécialistes des relations internationales la question des rapports entre Etats. Seule la prise en compte de cette logique interétatique dans une socio-histoire de l'immigration permet ici de saisir la complexité d'une séquence historique où la reproduction des pratiques administratives issues du moment colonial va de pair, dans d'autres lieux sociaux, avec une transformation des chaînes d'interdépendances dans lesquelles s'inscrivent des flux migratoires très anciens.

75 . Elias N., Engagement et distanciation, op. cit., p. 71 : «S'appuyant sur une longue tradition, les sociologues s'occupent essentiellement de problèmes relatifs à un plan particulier de la réalité sociale, celui qui se situe dans le cadre intérieur de l'Etat. [...] Les problèmes des rapports entre Etats par exemple ne sont pas considérés à proprement parler comme sociologiques. Classés comme politiques, ils sont rangés parmi les problèmes des relations internationales et donc renvoyés au domaine de recherche de la science politique ». 